Please do not remove this page

RMIT

UNIVERSITY

\title{
Crystallization kinetics of polydisperse colloidal hard spheres. II. Binary mixtures
}

Martin, Stephen; Bryant, Gary; Van Megen, William

https://researchrepository.rmit.edu.au/esploro/outputs/9921858569801341/filesAndLinks?institution=61RMIT_INST\&index=null

Martin, S., Bryant, G., \& Van Megen, W. (2005). Crystallization kinetics of polydisperse colloidal hard spheres. II. Binary mixtures. Physical Review E - Statistical, Nonlinear, and Soft Matter Physics, 71(2), 021404/1-021404/7. https://doi.org/10.1103/PhysRevE.71.021404

Published Version: https://doi.org/10.1103/PhysRevE.71.021404

Repository homepage: https://researchrepository.rmit.edu.au

(C)2005 The American Physical Society

Downloaded On 2023/04/27 01:19:00 +1000

Please do not remove this page 


\title{
Crystallization kinetics of polydisperse colloidal hard spheres. II. Binary mixtures
}

\author{
S. Martin, ${ }^{*}$ G. Bryant, ${ }^{\dagger}$ and W. van Megen ${ }^{\star}$ \\ Applied Physics, School of Applied Sciences, Royal Melbourne Institute of Technology, GPO Box 2476V, Melbourne 3001, Australia
}

(Received 3 November 2004; published 24 February 2005)

\begin{abstract}
In this paper we present measurements of the crystallization kinetics of binary mixtures of two different sized hard sphere particles. The growth of the Bragg reflections over time were analyzed to yield the crystallite scattering vector, the total amount of crystal, and the average linear crystal size. It was observed that a particle size distribution skewed to higher sized particles has a less detrimental effect on the crystal structure than a skew to smaller sized particles. In the latter case we observe that initial crystallite growth occurs at only a small number of sites, with further crystallization sites developing at later times. Based on these measurements we elaborate further on the previously proposed growth mechanism whereby crystallization occurs in conjunction with a local fractionation process in the fluid, which significantly affects the kinetic growth of crystallites in polydisperse systems.
\end{abstract}

DOI: 10.1103/PhysRevE.71.021404

PACS number(s): 64.70.Dv, 81.10.Fq, 82.70.Dd

\section{INTRODUCTION}

The occurrence of phase transitions in model hard sphere systems is now well established theoretically (e.g., Kirkwood [1] and Hoover and Ree [2]). Experimentally, colloidal particles suspended in an appropriate fluid are very useful systems for studying these phase transitions (e.g., Pusey and van Megen [3]). The size range of typical colloidal particles $(\sim 50-500 \mathrm{~nm})$ means that the length scales can be probed using visible light scattering techniques, and the time scales for crystallization and other phase transformations are long enough (minutes to days) to allow for detailed study. The colloidal hard sphere particles studied here can, under appropriate conditions, produce large (tens of microns) crystals which have Bragg spacings comparable to the wavelength of visible light. The kinetics of crystal growth can be studied using light scattering techniques, in particular laser light Bragg scattering, pioneered by Clark et al. [4] and Dhont et al. [5].

One of the main results to come out of recent work is that polydispersity has a significant influence on crystallization [6-12]. In a previous paper [13] we presented crystallization kinetics from two different lattices with differing particle size distributions (PSDs). It was found that although the particles had similar equilibrium phase behavior, the particles with a negative skewed PSD (i.e., with a size distribution skewed to smaller sizes) crystallized an order of magnitude more slowly than particles with a more symmetrical distribution. From these results we suggested a mechanism by which local fractionation [14] of the particles in the fluid can account for the slowing down of the solidification process in polydisperse samples. These results, and their interpretation, are consistent with recent molecular dynamics simulations

\footnotetext{
*Present address: Universität Konstanz, Fachbereich Physik, Fach M621, 78457 Konstanz, Germany. Electronic address: stephen.martin@uni-konstanz.de

†Electronic address: gary.bryant@rmit.edu.au

${ }^{\ddagger}$ Electronic address: bill.vanmegen@rmit.edu.au
}

[15]. Using a simple model polydisperse system (binary mixture with a size ratio of 0.9 ), these simulations showed that crystallization is dramatically slowed by the need for local compositional changes, and concluded that a glassy phase cannot exist for a monodisperse molecular dynamics system.

While our previous experimental work involved two lattices with inherently different polydispersities, here we study binary mixtures of those lattices. By adding progressively larger amounts of particles of slightly different size to the distribution, we investigate not only the effects of polydispersity, but also the differences between adding particles either larger than, or smaller than, the average particle size. The results of these investigations build on the idea that crystallization in polydisperse hard spheres is governed by a local fractionation process.

\section{METHOD}

The experimental methods and analysis used here have been described in detail previously [13] and will be summarized here only briefly. The particles used in this study consisted of a copolymer core of methylmethacrylate (MMA) and tri-fluoroethylacrylate (TFEA). To prevent coagulation of the particles, a stabilizing barrier of poly-12hydroxystearic acid (PHSA), about $10 \mathrm{~nm}$ thick, was chemically bonded to the surface. The refractive index of the particle core, stabilizing layer, and suspending solvent (cisdecalin; $n=1.483$ at $25^{\circ} \mathrm{C}$ ) are numerically close. Under these conditions the particle scattering form factors are very sensitive to the interplay between scattering from the core and the stabilizing layer, and thus the scattering is very sensitive to both the amount of TFEA in the core, and the temperature [16]. As in previous work [17], this feature is exploited to distinguish optically between the species in the mixtures. In this paper the partial structure factors were obtained by conducting the measurements at $24^{\circ} \mathrm{C}$ for latex W and $15{ }^{\circ} \mathrm{C}$ for latex $\mathrm{X}$. These temperatures provide the optimum ratio of scattering powers for the two lattices. The average core radius and effective polydispersity are $R_{\mathrm{X}}=300 \mathrm{~nm}$ and $6.7 \%$ for $\mathrm{X}$ and $R_{\mathrm{W}}=245 \mathrm{~nm}$ and $9-10 \%$ 
TABLE I. Composition of the binary sample mixtures studied. Composition of samples is given as the number fraction of larger (latex X) particles $x$, calculated from Eq. (1). Also tabulated are the partial volume fractions of each latex type in the sample.

\begin{tabular}{ccccc}
\hline \hline & $\begin{array}{c}\text { Volume } \\
\text { Fraction }\end{array}$ & & \multicolumn{2}{c}{ Partial volume fraction } \\
\cline { 4 - 5 } Sample & $(\phi)$ & $x$ & Latex W $\left(\phi_{\mathrm{W}}\right)$ & Latex X $\left(\phi_{\mathrm{X}}\right)$ \\
\hline $\mathrm{W} 2[0.54]$ & 0.5408 & 0.01 & 0.5292 & 0.0116 \\
$\mathrm{~W} 2[0.55]$ & 0.5507 & 0.01 & 0.5389 & 0.0118 \\
$\mathrm{X} 2[0.52]$ & 0.5202 & 0.988 & 0.0029 & 0.5176 \\
$\mathrm{X} 2[0.53]$ & 0.5303 & 0.988 & 0.0029 & 0.5274 \\
$\mathrm{X} 2[0.54]$ & 0.5400 & 0.988 & 0.0030 & 0.5370 \\
$\mathrm{X} 2[0.55]$ & 0.5530 & 0.988 & 0.0030 & 0.5500 \\
$\mathrm{X} 3[0.51]$ & 0.5121 & 0.974 & 0.0062 & 0.5059 \\
$\mathrm{X} 3[0.53]$ & 0.5304 & 0.974 & 0.0064 & 0.5240 \\
$\mathrm{X} 4[0.53]$ & 0.5304 & 0.897 & 0.0265 & 0.5039 \\
$\mathrm{X} 4[0.54]$ & 0.5401 & 0.897 & 0.0270 & 0.5131 \\
\hline \hline
\end{tabular}

for $\mathrm{W}$, with the latter being skewed to smaller sizes [18], so $R_{\mathrm{W}} / R_{\mathrm{X}} \sim 0.82$. Assuming a binary mixture of monodisperse hard spheres of the size ratio used here, an eutectic phase diagram is expected similar to that studied by Henderson [9]. Henderson also experimentally showed that these colloidal mixtures exhibit the expected demixing on solidification.

The individual particles have phase behavior compatible with that of a simple hard sphere system [3]. From sedimentation experiments the apparent melting and freezing volume fractions are identified, and then scaled to an effective hard sphere volume fraction by referencing the measured freezing volume fraction to the theoretical value of 0.495 . The effective melting volume fraction $\phi_{\text {melting }}$ can then be determined [19].

The compositions of mixtures of $\mathrm{X}$ and $\mathrm{W}$ are given by the number fraction $x$ of the larger particles:

$$
x=\frac{N_{\mathrm{X}}}{\left(N_{\mathrm{X}}+N_{\mathrm{W}}\right)},
$$

where $N_{\mathrm{X}}$ and $N_{\mathrm{W}}$ are the number concentrations of species $\mathrm{X}$ and $\mathrm{W}$. The compositions and nomenclature of the lattices studied are given in Table I. Not all binary mixtures were studied over the whole range of volume fractions $(\phi=0.52-0.55)$. The volume fractions missing were not attempted due to the finite amount of stock of the species $X$ and $\mathrm{W}$.

The time elapsed following mixing, $\tau=t / \tau_{b}$, is expressed in units of the Brownian time, $\tau_{b}=R^{2} / D_{\mathrm{o}}$, where $R$ is the average particle radius of the latex being measured (in the binary mixtures the radius of the major component is used) and $D_{\mathrm{o}}$ is the free particle diffusion coefficient for the same latex. Each experiment ran for approximately 2.7 days giving the final elapsed time, $\tau_{f}$, of $4 \times 10^{5}$ for latex $\mathrm{X}$ and $10^{6}$ for latex W. The range of values of $\phi$ and X listed in Table I are limited in part by the duration of the measurements, and in part by the limited amount of latex available.

Crystallization kinetics were measured using laser light Bragg scattering. In this method laser light scattered from the main interlayer reflection is collected from a scattering volume of $\sim 1 \mathrm{~cm}^{3}$ containing many seemingly randomly oriented crystallites. In this work the averaging over crystal orientations is improved further by averaging over the Debye-Scherrer cone [20]. Crystal growth is characterized from the measured structure factor $S(q, \tau)$, as a function of the scattering vector $q=(4 \pi n / \lambda) \sin (\theta / 2)$ and elapsed time $\tau$. Details of the analysis may be found in Ref. [13]. The parameters extracted from the data are

(1) The crystallinity $X(\tau)$, which is proportional to the amount of sample in the scattering volume which has been converted to a Bragg reflecting phase, and is determined by integrating the structure factor over the area of the main Bragg reflection:

$$
X(\tau)=\int S_{c}(q, \tau) d q
$$

(2) The peak maximum $q_{\max }(\tau)$, which is estimated from Gaussian fits to the peak of the structure factor, and is inversely proportional to the interlayer spacing.

(3) The average linear dimension of the crystals $L(\tau)$, which is determined from the full width at half maximum of the peak $\delta_{q}(\tau)$,

$$
L(\tau)=\frac{2 \pi K}{\delta q(\tau)},
$$

where $K=1.0747$ is the Scherrer constant for a spherical shaped crystal.

\section{RESULTS AND DISCUSSION}

\section{A. Final structure factors}

The initial and final structure factors (measured at times $\tau_{o}$ and $\tau_{f}$ ) for all lattices studied are shown in Fig. 1. Samples were also made with compositions of $x=0.08,0.33,0.51$, and 0.68 (data not shown). None of these samples showed any signs of crystallization at volume fractions in the coexistence range expected for such mixtures.

A number of features are apparent from Fig. 1. First, both W1 and W2 are similar, with evidence of only one peak, which we have previously interpreted as evidence of a lack of registration of planes [14] (although other interpretations may be possible, the kinetic parameters determined here are not model dependent). In contrast, the $\mathrm{X}$ latex shows the three peaks characteristic of random hexagonal close packing [21].

Second, it is observed for all samples that increasing the volume fraction suppresses the crystal structure. Third, in the W series of samples, it appears that on the inclusion of the second component (increasing $\mathrm{X}$ from 0 to 0.01 ), the structure becomes more like that of a one component sample at a volume fraction about 0.01 lower (i.e., the peak for W2[0.55] is similar in magnitude to that of W1[0.54]). For the two binary mixtures with $x=0.01$ the partial volume fractions of 

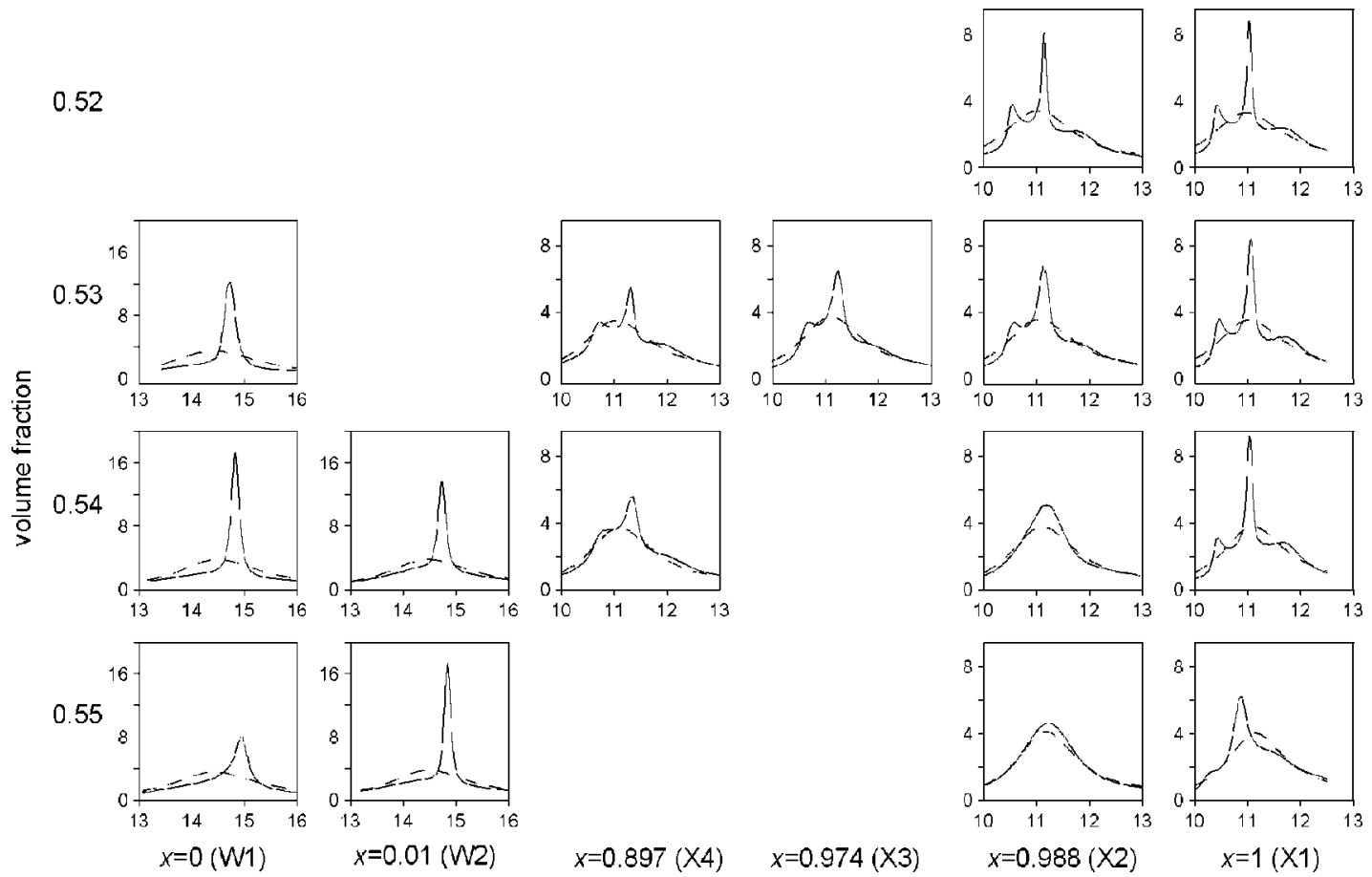

FIG. 1. Structure factors for all samples studied shown in the fluid phase (dashed line) at time $\tau=\tau_{o}$ and the final structure factor measured at the end of the experimental run (solid line) at time $\tau_{f}$. Note that $\tau_{o} \neq 0$, as experiments are begun after allowing the tumbled sample to stop flowing. The $x$ scale is the scattering vector $q$ in units $\mu \mathrm{m}^{-1}$.

the latex $\mathrm{W}$ component are $\phi_{\mathrm{W}}=0.53$ for $\mathrm{W} 2[0.54]$ and $\phi_{\mathrm{W}}$ $=0.54$ for $\mathrm{W} 2[0.55]$. This shows that the inclusion of this small amount of the second (larger) component has very little effect on the structure of the solid phase produced by the latex $\mathrm{W}$ particles.

Fourth, in contrast to the $\mathrm{W}$ series, the $\mathrm{X}$ series $(x \geqslant 0.897$, where smaller particles are being added to latex $\mathrm{X}$ ), the general trend is that the structure factor at the same volume fraction decreases in magnitude with decreasing $x$ (seen most clearly at $\phi=0.53$ ). Interestingly at $\phi=0.54$ or higher when $x=0.988$ (samples X2[0.54] and X2 [0.55]) the structure factor peak remains very broad and there is no evidence of the appearance of any crystal peaks (see later). In the case where the amount of smaller particles is increased by a factor of 10 (sample $\mathrm{X} 4[0.54]$ where $x=0.897$ ) the three crystal peaks again become visible. This unusual feature will be discussed later.

The positions of the main peak at the end of the experimental run $\left[q_{\max }\left(\tau_{f}\right)\right]$ are listed in Table II. The main observations here are that the addition of a small fraction of larger particles (W2) has marginally increased the lattice spacings [i.e., reduced $\left.q_{\max }(\tau)\right]$ between close-packed planes, though the structure remains unaffected (Fig. 2). In contrast, the addition of smaller particles $(x \geqslant 0.897)$ has caused the lattice spacings to decrease.

\section{B. Kinetics \\ 1. Small $x$}

The crystallization process, as quantified by $X(\tau), q_{\max }(\tau)$, and $L(\tau)$, is displayed for $\mathrm{W} 1$ and $\mathrm{W} 2$ in Figs. 2-4.
Figure 2 shows $\log X(\tau)$ vs $\log \tau$. The most obvious feature of these data is that adding a small fraction of larger particles slows the fluid-crystal conversion. At long times the W1[0.53] sample exhibits the plateau believed to correspond to the long time annealing stage [22]. For the other samples the experimental time is not long enough to reach the plateau (longer experiments are precluded by sedimentation effects). However, notwithstanding this, it appears that approximately the same proportion of sample crystallizes in all samples.

Figure 3 shows $q_{\max }(\tau)$ vs $\log \tau$. Although the type of structure is not changed by the inclusion of the second component, the peak positions clearly decrease as the second component is added. The initial contraction in the crystal lattice [i.e., the increase in $q_{\max }(\tau)$ ] observed for the single component samples appears to be nonexistent or much re-

TABLE II. Scattering vector of the peak position $q_{\max }$ of the interplanal reflection $[\mathrm{fcc}(111)]$ taken at the end of the experimental run, time $\tau_{f}$, for all samples. All values have an uncertainty of $\pm 0.04 \mu \mathrm{m}^{-1}$.

\begin{tabular}{ccccc}
\hline \hline & $\begin{array}{c}\phi=0.52 \\
q_{\max }\left(\tau_{f}\right) \\
\left(\mu \mathrm{m}^{-1}\right)\end{array}$ & $\begin{array}{c}\phi=0.53 \\
q_{\max }\left(\tau_{f}\right) \\
\left(\mu \mathrm{m}^{-1}\right)\end{array}$ & $\begin{array}{c}\phi=0.54 \\
q_{\max }\left(\tau_{f}\right) \\
\left(\mu \mathrm{m}^{-1}\right)\end{array}$ & $\begin{array}{c}\phi=0.55 \\
q_{\max }\left(\tau_{f}\right) \\
\left(\mu \mathrm{m}^{-1}\right)\end{array}$ \\
\hline W1 & & 14.74 & 14.83 & 14.92 \\
W2 & & & 14.74 & 14.85 \\
X1 & 11.04 & 11.05 & 11.04 & 10.86 \\
X2 & 11.15 & 11.14 & 11.22 & 11.36 \\
X3 & & 11.25 & & \\
X4 & & 11.30 & 11.34 & \\
\hline \hline
\end{tabular}




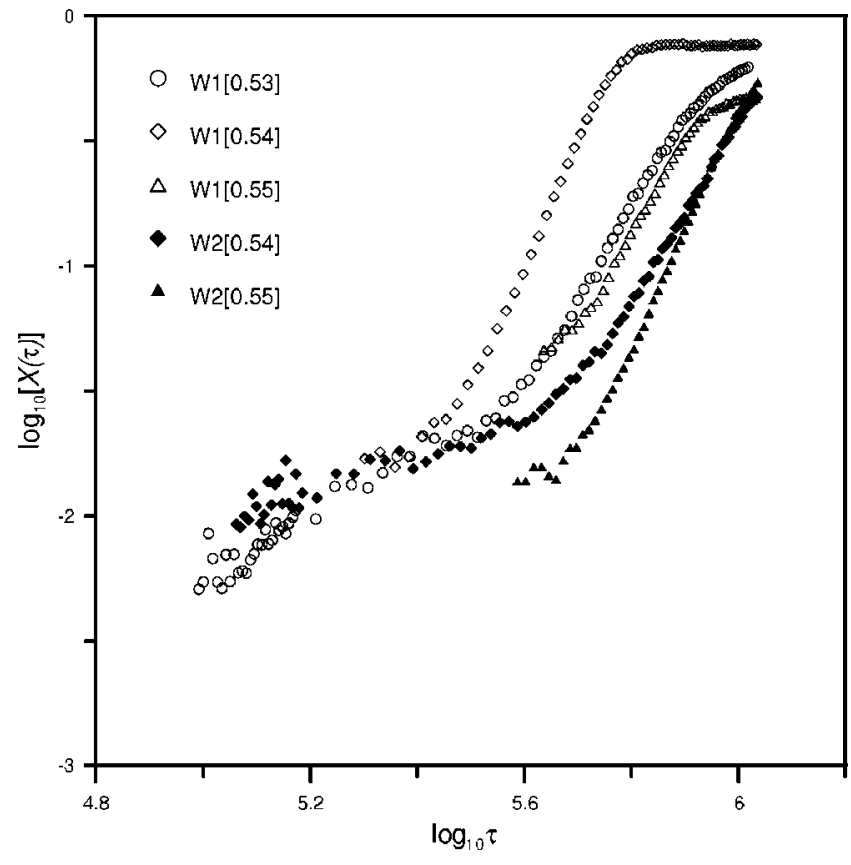

FIG. 2. Crystallinity $X(\tau)$ as a function of $\log$ reduced time for all samples composed of predominantly small particles $(x \leqslant 0.01)$.

duced for the W2 samples. Interestingly, $q_{\max }(\tau)$ for $\mathrm{W} 1[0.53]$ and $\mathrm{W} 2[0.54]$ are very similar, even decreasing at the same time (at $\log _{10} \tau \approx 5.8$ ) -i.e., the mixture behaves like the pure latex $\mathrm{W}$ sample at the same partial volume fraction. This is not so for $\mathrm{W} 1[0.54]$ and $\mathrm{W} 2[0.55]$; while they are similar in magnitude, there is a significant time delay in $\mathrm{W} 2[0.55]$ before there is an observed decrease in $q_{\max }(\tau)$.

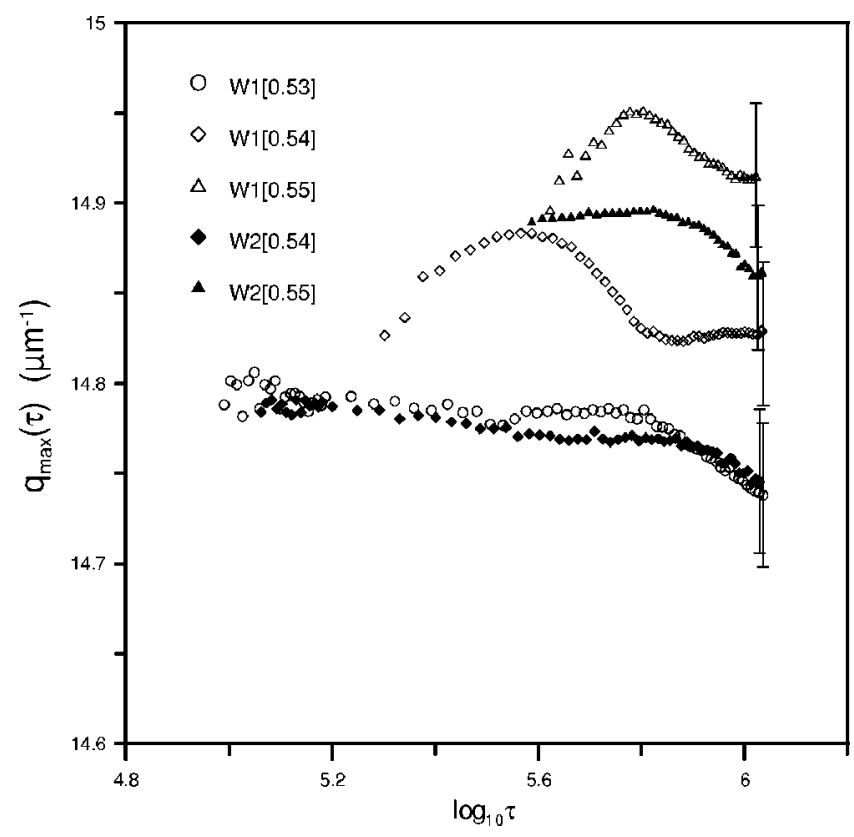

FIG. 3. Scattering vector of the position of the main reflection as a function of $\log$ reduced time for all samples composed of predominantly small particles $(x \leqslant 0.01)$. The final error bars show the absolute uncertainty due to calibration of the detector angle [13].

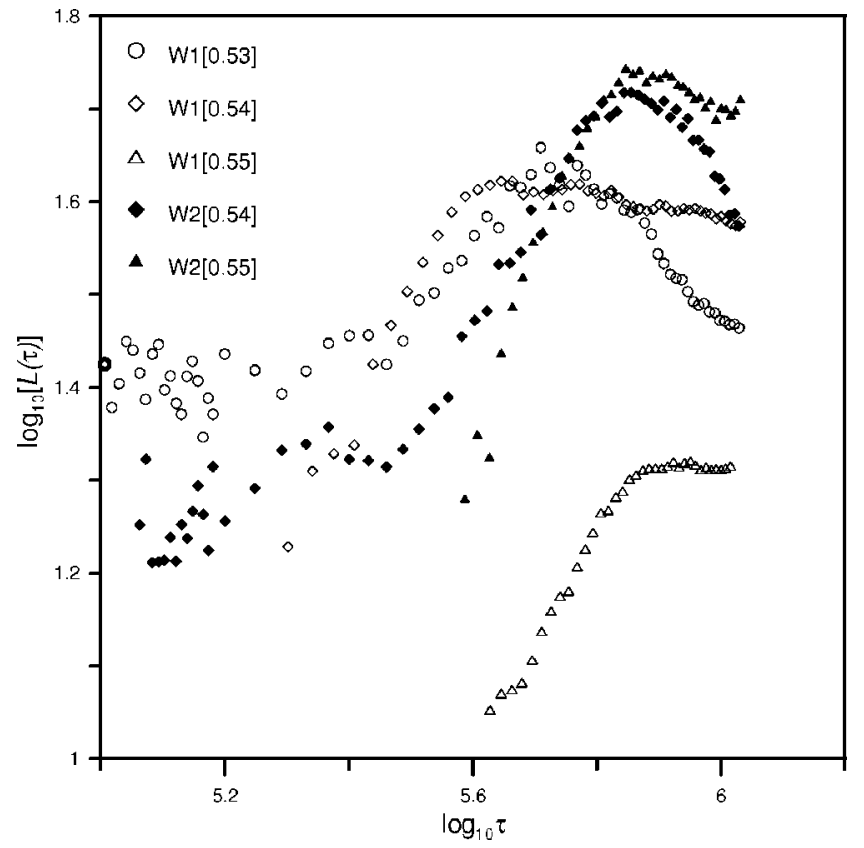

FIG. 4. Average linear crystal dimension $L(\tau)$ as a function of time for all samples composed of predominantly small particles $(x \leqslant 0.01)$.

Figure 4 shows the log of the average linear crystallite dimension $L(\tau)$ vs elapsed time. Interestingly, this indicates that the crystallites grow to a larger average size for the $x=0.01$ samples than for the $x=0$ samples at the same total volume fraction. The larger average size observed for the $x=0.01$ samples may be due to the fact that the need for segregation leads to fewer growing crystallites, which are then able to grow larger, on average. Note that in contrast to the $q_{\max }(\tau)$ data, the $L(\tau)$ for $\mathrm{W} 1[0.53]$ and $\mathrm{W} 2[0.54]$ are qualitatively different.

Summarizing the effect of adding a second larger component, we observe that (i) conversion of fluid to crystallite is slowed (Fig. 2); (ii) the lattice spacing increases (Fig. 3); and the average linear dimension increases (Fig. 4).

\section{Large $x$}

For the samples composed predominantly of larger particles $(x \geqslant 0.897), X(\tau)$ and $L(\tau)$ are shown in Figs. 5 and 6, respectively. For brevity $q(\tau)$ is not shown as it does not provide any extra information.

At intermediate volume fractions $(0.52 \leqslant \phi \leqslant 0.54)$, as $x$ increases, there is an increasing delay in the onset of rapid crystallization (shown as the line designated $\mu$ in Fig. 5). In addition, at volume fractions of 0.53 and 0.54 the mixtures exhibit an initial slow conversion region (designated $\mu_{\mathrm{i}}$ in the figure). For pure latex $\mathrm{X}$ this region is only seen at 0.55 , though it is seen at all volume fractions for latex W (Fig. 2). Looking at $\phi=0.53$, where all sample compositions of $x$ were studied, the onset time for crystallization increases by more than an order of magnitude as the amount of the second component is increased.

At the highest volume fraction studied, $\phi=0.55$, the inclusion of only $1.2 \%$ of the smaller particles completely sup- 

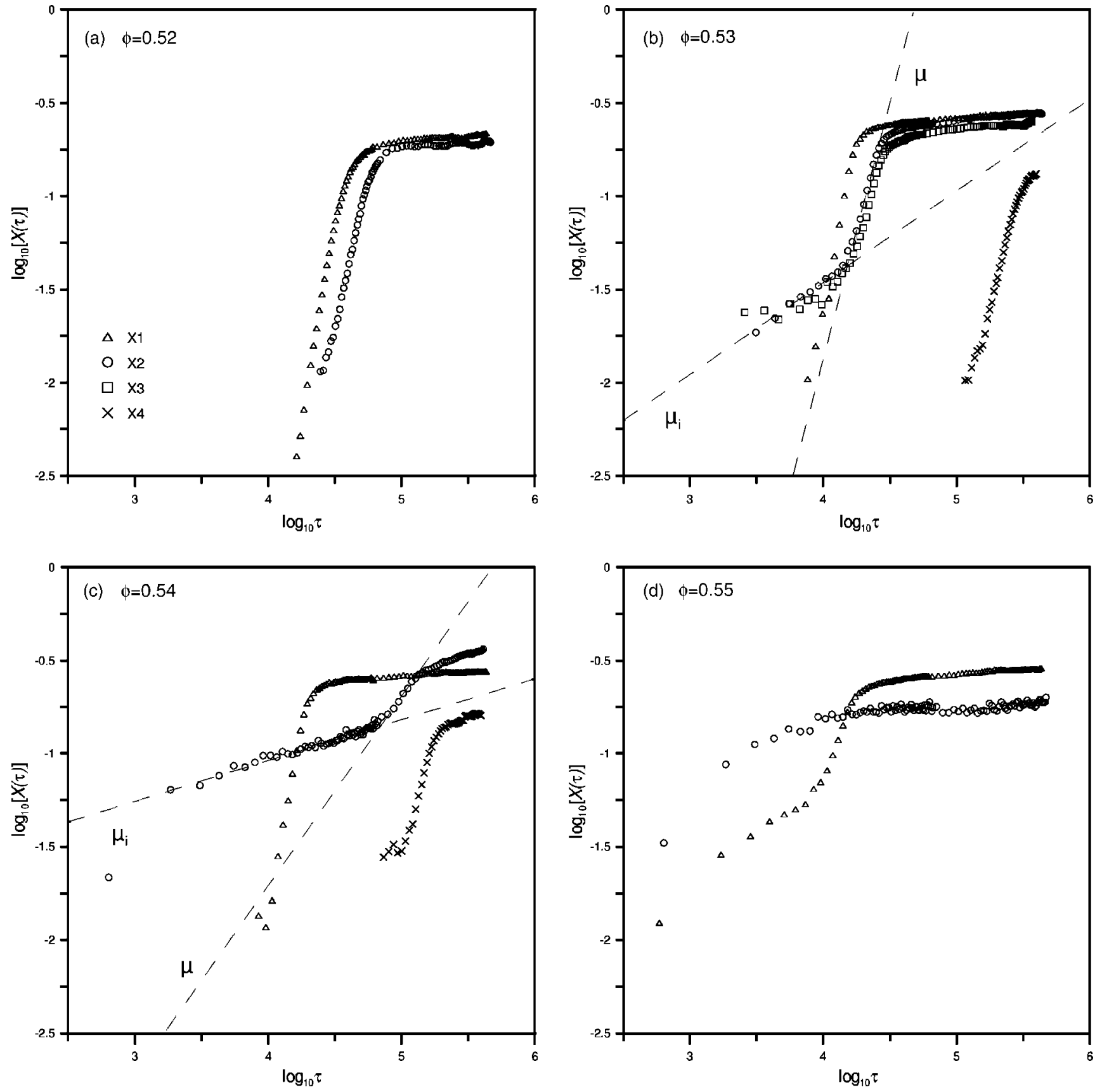

FIG. 5. Crystallinity $X(\tau)$ as a function of log reduced time for samples composed of predominantly large particles $(x \geqslant 0.897)$ at volume fractions (a) $\phi=0.52$, (b) $\phi=0.53$, (c) $\phi=0.54$, and (d) $\phi=0.55$. All data have been normalized to the final value of the X1[0.55] sample. The slope of the dashed lines are the growth coefficients of rapid conversion $\mu$ and the initial period $\mu_{\mathrm{i}}$ as referred to in the text.

presses crystallization (see Fig. 1). However, $X(\tau)$, determined from the difference between the measured structure factors at times $\tau_{f}$ and $\tau_{o}$, does evolve, increasing by half an order of magnitude. In contrast, the measured $L$ (Fig. 6), is virtually constant. Thus, while no Bragg reflections evolve, either visually or instrumentally, it appears that the metastable fluid is undergoing significant intermediate range reordering.

For the lower volume fractions $L(\tau)$ exhibits a period of rapid growth followed by a later period of slow growth (Fig. 6). For 0.53 and 0.54 the addition of the second component also results in a period of slow crystal growth, which pre- cedes the period of rapid growth. This is also seen for $\mathrm{X} 1[0.55]$. For X2[0.54] the length of time for which the initial slope $\mu_{\mathrm{i}}$ is observed dominates most of the crystallization process, showing only a slight increase in average crystallite size in time. This can be explained in terms of an ongoing fractionation process, as described previously [13]. With the inclusion of $\approx 10 \%$ smaller particle, as in the two X4 samples, the onset of crystallization is delayed by an order of magnitude. At earlier times, no reliable fits can be made to the data.

To summarize, with the samples composed predominantly of the larger particles, the inclusion of the smaller latex 

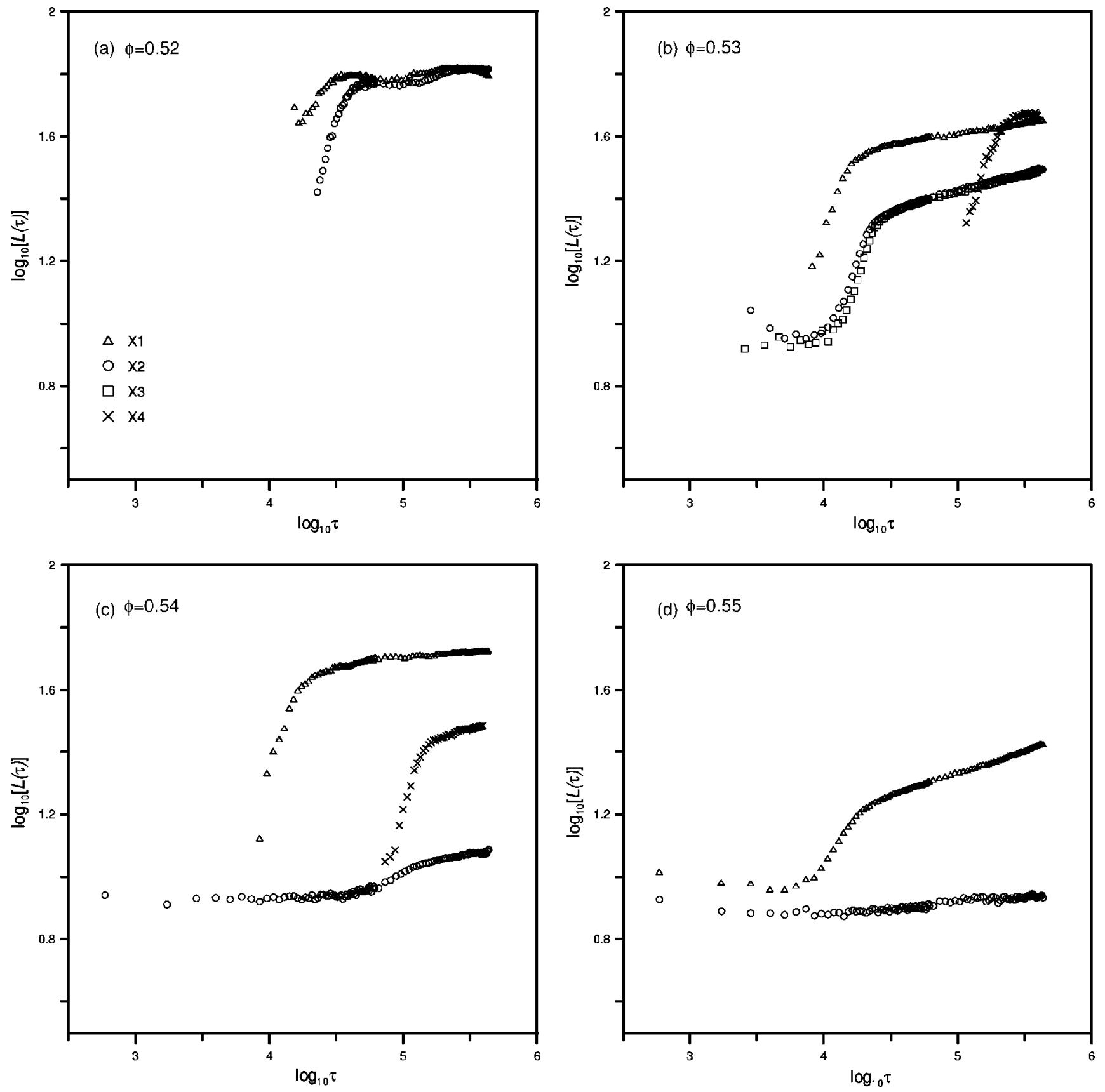

FIG. 6. Average crystallite size $L(\tau)$ as a function of log reduced time for samples composed of predominantly large particles $(x \geqslant 0.897)$ at volume fractions (a) $\phi=0.52$, (b) $\phi=0.53$, (c) $\phi=0.54$, and (d) $\phi=0.55$.

caused a slowing of the crystallization process. When only a small number (1-2\%) was added, an increase was observed in the initial period of slow growth $\mu_{\mathrm{i}}$, which we attribute to a fractionation process. The inclusion of the smaller particles suppressed the rapid formation of crystallites, extending the amount of time that is needed to filter the fluid to provide suitable particles for inclusion in the growing crystallites. At higher volume fractions, where rapid crystallization is evident in the one component sample, adding the smaller latex increased the fractionation period dramatically, and prevented the formation of large crystallites.

\section{CONCLUSION}

Changing the shape of the particle size distributions through the production of binary mixtures with a size ratio near 1 , lends support to the conclusions drawn in our previous work on single component polydisperse systems. Kinetically the addition of a small fraction of larger particles causes a slowing of the crystallization process. Structurally, the fact that the mixture has a structure factor similar to that of the one component system at the same partial volume fraction of $\mathrm{W}$ suggests that the larger particles are largely fractionated out. 
The addition of smaller particles, on the other hand, increased the onset time for crystallization, and in some cases two growth regimes were evident, as seen for the skewed "single component" distributions in our previous work.

From the data presented, it is seen that a particle size distribution skewed to higher sized particles has a less detrimental effect on the crystal structure than a skew to smaller sized particles. A small sized skewness structurally retards large crystallite growth at higher volume fractions. There are still a large number of initial nucleation sites, but crystal growth is limited as suitable sized particles in the neighboring fluid are scarcer. With a larger negative skewness in the PSD the initial conditions of the fluid mean there are fewer initial nucleation sites, so only a few sites grow at first, with further nucleation sites developing at later times when the fluid becomes more fractionated.

Regardless of how the size distribution is skewed, it is observed that an increase in polydispersity will increase the delay time for the onset of crystallization as more fractionation of the fluid is required before crystallization can proceed. Trends relating to the effect of volume fraction on nucleation rates are not so easily resolved from these experiments, and further experiments are underway to explore these effects.

The evidence presented here and in related work demonstrate that the effects of polydispersity are complex, and are very sensitive to the detailed nature of the particle size distribution. This suggests that the concept of a simple limiting polydispersity, based on the Lindemann melting criterion [23], may need to be reexamined.
[1] J. G. Kirkwood, J. Chem. Phys. 7, 919 (1939).

[2] W. G. Hoover and F. H. Ree, J. Chem. Phys. 49, 3609 (1968).

[3] P. N. Pusey and W. van Megen, Nature (London) 320, 340 (1986).

[4] N. A. Clark, A. J. Hurd, and B. J. Ackerson, Nature (London) 281, 57 (1979).

[5] J. K. G. Dhont, C. Smits, and H. N. W. Lekkerkerker, J. Colloid Interface Sci. 152, 386 (1992).

[6] S. I. Henderson and W. van Megen, Phys. Rev. Lett. 80, 877 (1998).

[7] P. N. Pusey, in Liquids, Freezing and the Glass Transition, edited by J. P. Hansen, D. Levesque, and J. Zinn-Justin (NorthHolland, Amsterdam, 1991) p. 763.

[8] D. A. Kofke and P. G. Bolhuis Phys. Rev. E 59, 618 (1999).

[9] S. Henderson, Ph.D. thesis, Department of Applied Physics, Royal Melbourne Institute of Technology, Melbourne, 1999, p. 117.

[10] P. Bartlett, J. Phys.: Condens. Matter 12, A275 (2000).

[11] R. M. L. Evans and C. B. Holmes, Phys. Rev. E 64, 011404 (2001).

[12] S. Auer and D. Frenkel, Nature (London) 413, 711 (2001).

[13] S. Martin, G. Bryant, and W. van Megen, Phys. Rev. E 67,
061405 (2003)

[14] S. Martin, G. Bryant, and W. van Megen, Phys. Rev. Lett. 90, 255702 (2003).

[15] S. R. Williams, I. K. Snook, and W. van Megen, Phys. Rev. E 64, 021506 (2001).

[16] S. M. Underwood and W. van Megen, Colloid Polym. Sci. 274, 1072 (1996).

[17] S. Williams and W. van Megen, Phys. Rev. E 64, 041502 (2001).

[18] G. Bryant, S. Martin, B. Budi, and W. van Megen, Langmuir 19, 616 (2002).

[19] B. J. Ackerson, S. E. Paulin, B. Johnson, W. van Megen, and S. M. Underwood, Phys. Rev. E 59, 6903 (1999).

[20] P. Francis, S. Martin, G. Bryant, W. van Megen, and P. A. Wilksch, Rev. Sci. Instrum. 73, 3872 (2002).

[21] P. N. Pusey, W. van Megen, P. Bartlett, B. J. Ackerson, J. G. Rarity, and S. M. Underwood, Phys. Rev. Lett. 63, 2753 (1989).

[22] J. L. Harland and W. van Megen, Phys. Rev. E 55, 3054 (1997).

[23] P. N. Pusey, J. Phys. (France) 48, 709 (1987). 